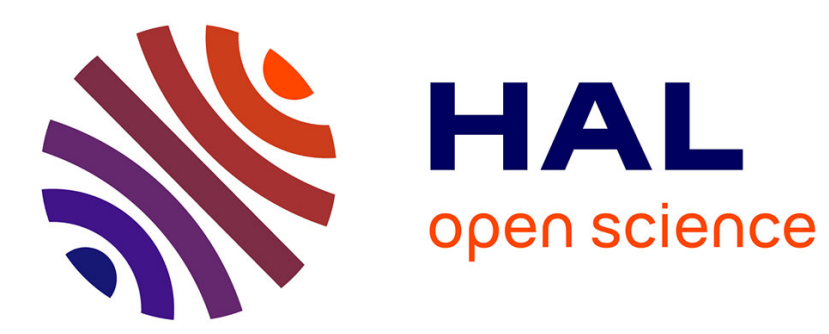

\title{
Geometry and spin-multiplicity of half-sandwich type transition-metal-benzene complexes
}

F. Rabilloud

\section{To cite this version:}

F. Rabilloud. Geometry and spin-multiplicity of half-sandwich type transition-metal-benzene complexes. Journal of Chemical Physics, 2005, 122 (13), pp.134303. 10.1063/1.1867434 • hal-03510534

\section{HAL Id: hal-03510534 \\ https://hal.science/hal-03510534}

Submitted on 13 Jan 2022

HAL is a multi-disciplinary open access archive for the deposit and dissemination of scientific research documents, whether they are published or not. The documents may come from teaching and research institutions in France or abroad, or from public or private research centers.
L'archive ouverte pluridisciplinaire HAL, est destinée au dépôt et à la diffusion de documents scientifiques de niveau recherche, publiés ou non, émanant des établissements d'enseignement et de recherche français ou étrangers, des laboratoires publics ou privés. 


\title{
Geometry and spin-multiplicity of half-sandwich type transition-metal-benzene complexes
}

\author{
F. Rabilloud ${ }^{\text {a) }}$ \\ Laboratoire de Spectrométrie Ionique et Moléculaire, UMR 5579 (Université Claude Bernard Lyon 1 \\ \& CNRS), 43 bd du 11 novembre 1918, 69622 Villeurbanne Cedex, France
}

(Received 14 October 2004; accepted 13 January 2005; published online 4 April 2005)

\begin{abstract}
The low lying electronic states of $3 \mathrm{~d}$ transition-metal-benzene complexes $\mathrm{MBz}$ (with $\mathrm{M}=\mathrm{Sc}, \mathrm{V}$, and $\mathrm{Ni}$ ) have been investigated by performing complete active space self-consistent field and multireference configuration interaction calculations. Geometries, energetics, and electronic structure are presented and discussed. The results concerning both the geometry and the spin multiplicity of the ground-state contrast with those obtained from previous calculations based on density functional theory. The disagreements between single-reference-based approaches and multireference methods in the characterization of neutral 3d-metal complexes are discussed. () 2005 American Institute of Physics. [DOI: 10.1063/1.1867434]
\end{abstract}

\section{INTRODUCTION}

The advent of the laser vaporization method has caused rapid progress in the study of metal-ligand molecules in the gas phase, and makes it possible to obtain fundamental information on the chemical and physical properties of complexes. Recently, the $3 d$-transition-metal-benzene $\mathrm{M}_{\mathrm{n}} \mathrm{Bz}_{\mathrm{m}}$ complexes were synthesized in the gas phase by Kaya and co-workers. ${ }^{1,2}$ These complexes were analyzed using mass spectroscopy, and their structures are predicted to be either sandwich or rice-ball configuration, depending on the transition metal. Early 3d-elements $(\mathrm{Sc}, \mathrm{Ti}$, and $\mathrm{V}$ ) form a multiple-decker sandwich structure $(\mathrm{n}, \mathrm{m}=\mathrm{n}+1$ compounds) in which metal atoms and benzene are alternatively piled up in one-dimensional structure, while late transition metals $(\mathrm{Fe}$, $\mathrm{Co}$, and $\mathrm{Ni}$ ) form a rice-ball structure in which central metal clusters are fully covered by benzene molecules. The stability of the sandwich structure has been confirmed for $\mathrm{V}_{\mathrm{n}} \mathrm{Bz}_{\mathrm{n}+1}^{+}$ complexes by ion mobility measurements and collisioninduced dissociation experiments, ${ }^{3}$ and also for a series of transition-metal $\mathrm{Bz}_{2}$ by electric dipole measurements. ${ }^{4}$

From the theoretical point of view, the energetics and geometries of the complexes of benzene with $3 \mathrm{~d}$-metal ions have been investigated using modified coupled-pair functional (MCPF) type calculations, ${ }^{5}$ and also with density functional theory (DFT) type calculations. ${ }^{6-9}$ Very recently, Kambalapalli and Ortiz ${ }^{10}$ have determined the vertical electrondetachment energies of $\mathrm{ScBz}^{-}$at the Møller-Plesset perturbation (MP2) + coupled-cluster with single, double, and perturbative triple excitations $(\operatorname{CCSD}(\mathrm{T}))$ level. However, few studies are available on neutral complexes. Yasuike and Yabushita ${ }^{11}$ have investigated the bonding scheme between benzene and metal atoms ( $\mathrm{Ti}, \mathrm{V}$, and $\mathrm{Cr}$ ) in sandwich structures by quantum chemical calculations. Froudakis, Andriotis and Menon, ${ }^{12}$ using tight-binding molecular dynamics simulations, have found a contrasting behavior for $\mathrm{Ni}$ and $\mathrm{V}$ in

\footnotetext{
a) Author to whom correspondence should be addressed. Fax: (33) 4-72431507. Electronic mail: franck.rabilloud@lasim.univ-lyon1.fr
}

their interaction with the benzene molecule. Finally, Rao and Jena ${ }^{13,14}$ have performed global optimization of the geometry of small $\mathrm{Ni}$ clusters interacting with benzene molecule, yielding equilibrium structures where the $\mathrm{Ni}$ atoms were found to be caged between the benzene molecules.

While various theoretical works have been concerned with the properties of small clusters $\mathrm{M}_{n} B z_{m}$ (with $n+m \geqslant 3$ ), very few results exist on $\mathrm{MBz}$ molecules. Yasuike and co-workers ${ }^{15}$ have calculated the potential energy curves for $\mathrm{M}-\mathrm{Bz}(\mathrm{M}=\mathrm{Ti}, \mathrm{V}$, and $\mathrm{Cr})$ using a full valence configurational interaction method with configuration-averaged selfconsistent-field (SCF) orbitals. Pandey and co-workers ${ }^{16}$ have investigated the electronic structure of transition-metalbenzene complexes using DFT/BPW91 type calculations. Despite some theoretical efforts, the fundamental electronic state of these molecules is not fully characterized yet. In particular, the spin multiplicity of the ground state is difficult to determine and different theoretical approaches may lead to controversial results. In a recent study, ${ }^{17}$ Pandey and coworkers shown that DFT/BPW91 type calculations lead to contradictory results concerning the ground-state spin multiplicity depending on the basis set and the pseudopotential used. The electronic calculations on transition-metal elements pose a considerable theoretical challenge. ${ }^{18}$ Due to the open d-shell structure, transition-metal systems have many spin-multiplet symmetries lying within a narrow energy range. For such systems, the single-reference-based approaches, such as $\operatorname{CCSD}(\mathrm{T})$ or DFT are well known to fail to determine the correct ground-state spin multiplicity and often favor states with highest spin multiplicities. Multireference techniques where electronic wave functions are treated as multiconfigurational functions are more suitable for the characterization of the multiplicity of the ground state. Unfortunately, as is well known, those calculations are computer intensive, and consequently many previous works were performed within the DFT formalism.

In this paper, we present the first ab initio study using multireference techniques to characterize the electronic states 
of transition-metal-benzene $\mathrm{MBz}$ complexes with $\mathrm{M}=\mathrm{Sc}, \mathrm{V}$, and Ni. In Sec. II we give details of the calculations, and in Sec. III we present the potential energy profile of the electronic ground state and some lowest-lying excited states having various spin or spatial symmetries for the three molecules VBz, ScBZ, and NiBz. Finally, in Sec. IV we discuss the geometry and the spin multiplicity of the ground state and compare our results with those from previous theoretical and experimental works.

\section{COMPUTATIONAL METHODS}

Calculations have been carried out by use of the computational chemistry program MOLPRO (Ref. 19) and the graphical user interface Gabedit. ${ }^{20}$ Metal and carbon atoms were represented through relativistic effective core pseudopotentials developed by Preuss and co-workers ${ }^{21,22}$ replacing ten core electrons on metal atoms and two core electrons on carbon. We occasionally used pseudopotentials developed by Stevens et al. ${ }^{23,24}$ but as the results were found to be very similar, we will only discuss results obtained from calculations performed with pseudopotentials of Preuss et al. The Gaussian basis sets used for metal atoms were (8s7p6dlf) contracted in [6s5p3dlf] from Ref. 21, which for carbon was $[4 \mathrm{~s} 2 \mathrm{p}]$ while for hydrogen we used a double zeta polarization basis as displayed in MOLPRO.

As explained above, a multireference technique is suitable to characterize the electronic ground state and the lowest-lying excited states of $\mathrm{M}-\mathrm{Bz}$ species. In such a sense, calculations have been performed through a complete active space self-consistent field (CASSCF) method and a multireference configuration interaction (MRCI) approach using the CASSCF configuration space as reference. The potential energy profiles were calculated at CASSCF level. The active space included the metal $4 s, 3 d, 5 s, 4 d$ orbitals for calculations on $\mathrm{NiBz}$ and $\mathrm{VBz}$ complexes. For $\mathrm{ScBz}$, the metal $4 p$ orbitals were added in the active space. The inner orbitals of metal and orbitals of benzene molecule were kept inactive in the CAS but were allowed to relax in the SCF process. For the geometry optimization we varied only the distance between the metal atom and the center of mass in the benzene molecule. Namely, the benzene molecule structure was not allowed to relax and was fixed to the isolated one optimized from a CASSCF calculation. Then a single-point MRCI (single and double excitations + Davidson correction) type of calculation was performed at the CASSCF equilibrium geometry for the ground state for each spin multiplicity in order to determine the binding energies and the dipole moments. For MRCI calculation, basis sets were further augmented by diffuse functions on both metal atom and benzene molecule, though this extension occurred to yield only a very small improvement.

\section{RESULTS}

Many previous studies have found that the transitionmetal-benzene complexes present a half-sandwich type structure in which the metal atom is generally located on the $\mathrm{C}_{6 \mathrm{v}}$ axis. ${ }^{5-10,15-17}$ Although some investigations on charged complexes occasionally found structures slightly distorted from the $\mathrm{C}_{6 \mathrm{v}}$ symmetry where the benzene ligand distorts to boat conformations, all the studies concerning the neutral systems yielded to either a perfect $\mathrm{C}_{6 \mathrm{v}}$ symmetry structures or a quasi- $\mathrm{C}_{6 \mathrm{v}}$ with a small deformation due to a very small JahnTeller effect. ${ }^{10,15-17}$ In the present work, calculations were performed in the $\mathrm{C}_{2 \mathrm{v}}$ point group but the $\mathrm{C}_{6 \mathrm{v}}$ symmetry of the $\mathrm{MBz}$ complex was maintained throughout. However, we occasionally performed a local optimization (in $\mathrm{C}_{2 \mathrm{v}}$ point group) to investigate the rigidity of benzene molecule. It was the case for the short-range state of $\mathrm{NiBz}$, for which the distance between metal atom and benzene molecule was found to be shorter than it was for the other complexes and so the possible distortion of the benzene molecule was expected to be bigger. We found only a small distortion: the $\mathrm{C}-\mathrm{C}$ and $\mathrm{C}-\mathrm{H}$ distances were found to be 1.405 and $1.068 \AA$, respectively, to be compared with the values of 1.386 and $1.071 \AA$, respectively, for isolated benzene. The $\mathrm{C}_{6 \mathrm{v}}$ symmetry of the complex was in fact not broken but hydrogen atoms were found to move from their initial position to a plane parallel to the carbon ring and located $0.021 \AA$ below it, and then more distant from the atom metal located above the carbon ring. Nevertheless, the deformation was not significant for the present study and the $\mathrm{C}_{6 \mathrm{v}}$ symmetry constraint with a rigid ring for benzene appeared to be appropriated. The possibility of the existence of the asymmetric-top molecules was also investigated for both $\mathrm{NiBz}$ and $\mathrm{ScBz}$ complexes. Local optimizations with the metal atom placed above the benzene plane but displaced from the center axis $\left(\mathrm{C}_{\mathrm{s}}\right.$ symmetry) were found to converge either in a perfectly symmetric structure or in dissociation into $\mathrm{Bz}+$ metal atom. Then, in the present calculation of the potential energy curves, we changed only the $\mathrm{M}-\mathrm{Bz}$ distance, the metal atom being located on the $\mathrm{C}_{6 \mathrm{v}}$ axis of the fixed benzene molecule.

Before showing the potential energy profile of the lowerlying states, it is useful to review the bonding scheme in transition-metal-benzene systems. The valence configuration of the isolated transition-metal atoms which are considered in the present study is $3 \mathrm{~d}^{\mathrm{n}} 4 \mathrm{~s}^{2}$ with the $3 \mathrm{~d}$ orbitals split into $3 \mathrm{da}_{1},\left(\mathrm{~d}_{z 2}\right), 3 \mathrm{de}_{1}\left(\mathrm{~d}_{\mathrm{xz}}\right.$ and $\left.\mathrm{d}_{\mathrm{yz}}\right)$, and $3 \mathrm{de}_{2}\left(\mathrm{~d}_{\mathrm{x} 2-\mathrm{y} 2}\right.$ and $\left.\mathrm{d}_{\mathrm{xy}}\right)$ in a $\mathrm{C}_{6 \mathrm{v}}$ symmetry. For the lower-lying states of $\mathrm{MBz}$ complex, the ligand orbitals will not be placed between the metal orbitals, and the order of valence orbitals will be $\mathrm{a}_{1}, \mathrm{e}_{1}, \mathrm{e}_{2}$ (from $\sigma$ and $\pi$ occupied orbitals of benzene), and $4 \mathrm{~s} / 3 \mathrm{~d}$ (partially filled from transition-metal atom). As the 4s orbital has a repulsive interaction with the doubly occupied benzene $a_{1}$ orbitals, the molecular diabatic state built from the $3 \mathrm{~d}^{\mathrm{n}} 4 \mathrm{~s}^{2}$ ground-state atomic configuration is repulsive. On the contrary, an excited atomic state having a $3 \mathrm{~d}^{\mathrm{n}+2}$ configuration has an attractive interaction with the benzene molecule, resulting in a stabilization of energy in a diabatic curve. As illustrated in Fig. 1, these two diabatic potential energy curves intersect each other resulting in an adiabatic ground state which can be either stable or metastable with respect to the dissociation into metal atom + benzene molecule, depending on the relative position of the diabatic curves. In all cases, the electronic configuration at the minimum will be primarily $3 \mathrm{~d}^{\mathrm{n}+2}$ or $3 \mathrm{~d}^{\mathrm{n}+1} 4 \mathrm{~s}^{1}$ while that at the dissociation limit will be $3 \mathrm{~d}^{\mathrm{n}} 4 \mathrm{~s}^{2}$. In the case of a metastable state, the 

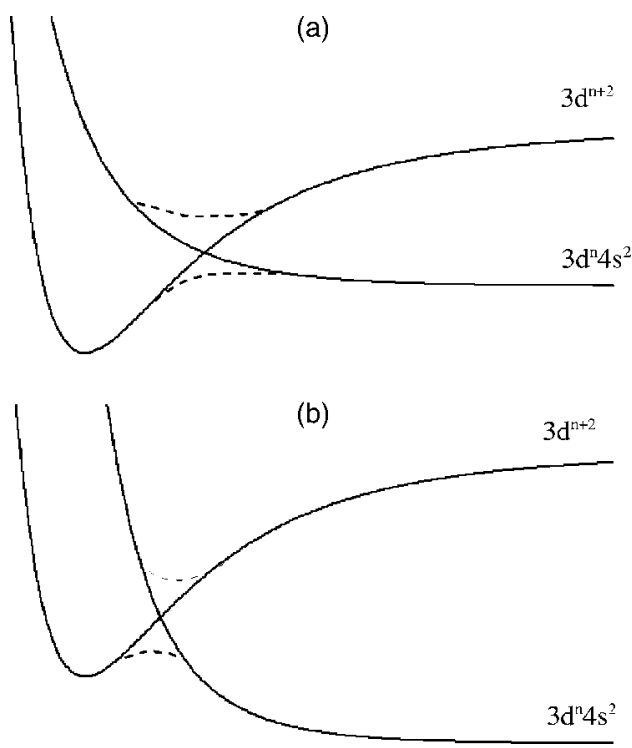

FIG. 1. Schematic representation of diabatic (full lines) and adiabatic (dashes) potential energy curves. The adiabatic ground state is either stable (case a) or metastable (case b) with respect to the dissociation in metal atom + benzene molecule.

minimum is above the asymptote corresponding to the metal atom + the benzene molecule in their respective ground states.

\section{A. VBz molecule}

The above bonding scheme is observed in the results of our calculations for VBz. At short range, the ground state for $\mathrm{VBz}$ is found to have a quartet multiplicity and to be metastable with respect to the dissociation into $\mathrm{Bz}+\mathrm{V}$ with an energy barrier of $0.11 \mathrm{eV}$ (see Fig. 2). An avoided crossing involving two diabatic states with a primarily $3 d^{4} 4 s^{1}+3 d^{5}$ and $3 \mathrm{~d}^{3} 4 \mathrm{~s}^{2}$ electronic configuration occurs within the 2.0-2.3 $\AA$ of $\mathrm{V}-\mathrm{Bz}$ distance. It results in metastable state having a primarily $3 d^{4} 4 s^{1}$ configuration at short range and a $3 d^{3} 4 s^{2}$ asymptote. Several excited states are shown in the Fig. 2 with, respectively, $3 \mathrm{~d}^{4} 4 \mathrm{~s}^{1}, 3 \mathrm{~d}^{3} 4 \mathrm{~s}^{2}$ and $3 \mathrm{~d}^{4} 4 \mathrm{~s}^{1}$ asymptotes. Close to the dissociation limit $(20 \AA)$, their relative energies from the ground state are $1.03,1.37$, and $1.77 \mathrm{eV}$, respectively, in very good agreement with the experimental atomic

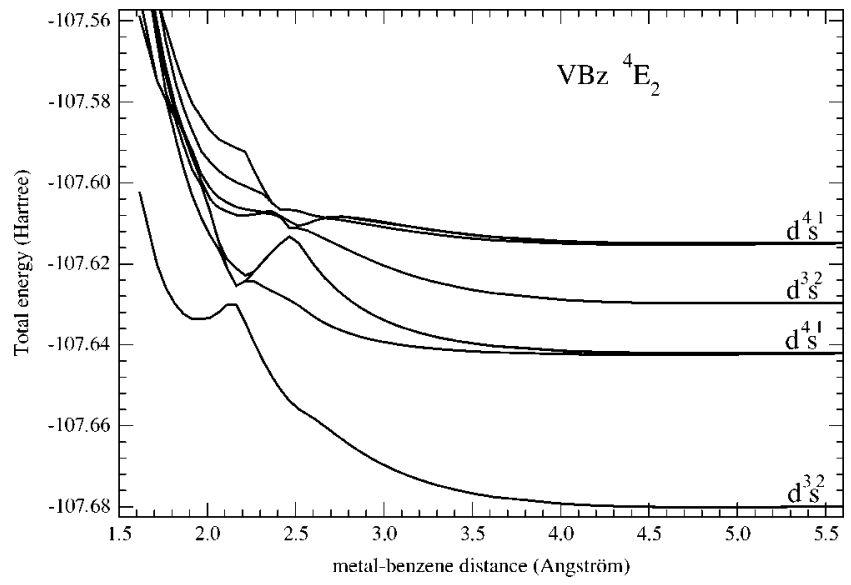

FIG. 2. Potential energy curves for the ${ }^{4} \mathrm{E}_{2}$ states for the VBz molecule. excitations of $1.04,1.18$, and $1.77 \mathrm{eV}$, respectively. In fact, at long range, within 4-5 $\AA$ for the metal-benzene distance, some molecular states are found to be slightly bound. The ground state of $\mathrm{VBz}$ is found to be bound with a $\mathrm{V}-\mathrm{Bz}$ distance of $4.82 \AA$ and with a binding energy of $350 \mathrm{~K}$. This state lies $1.26 \mathrm{eV}$ below the well displayed at short range and situated above the dissociation limit. The doublet and sextet spin multiplicities are also investigated. All results are given in Table I. At long range, a sextet, with $4 \mathrm{~s}^{1} 3 \mathrm{de}_{2}{ }^{2} 3 \mathrm{de}_{1}^{2}$ electronic configuration, is found to be bound. It lies $0.60 \mathrm{eV}$ above the quartet ground state and has a binding energy of $520 \mathrm{~K}$. At short range, a doublet state is found lying $0.44 \mathrm{eV}$ above the quartet while the sextet is found to be completely repulsive. These results are in very good agreement with those of the ab initio study by Yasuike et al. ${ }^{15}$ using a full valence configuration interaction method with configurationaveraged SCF orbitals.

\section{B. ScBz molecule}

The active space for the CASSCF calculations included the metal $4 s, 3 d, 5 s, 4 d, 4 p$ orbitals, the addition of the $4 p$ orbitals being proved useful to investigate the potential energy curves due to the important role of the $3 \mathrm{~d} 4 \mathrm{~s} 4 \mathrm{p}$ configurations in the atomic spectra of Sc. Both doublet and quartet spin multiplicities have been investigated with various spatial symmetries. Potential energy curves are drawn in Fig. 3 for the ${ }^{2} E_{2}$ states. The ground state is found to have a ${ }^{2} E_{2}$ symmetry and to be bound at long range with a metalbenzene distance of $4.96 \AA$ and with a binding energy of 260 $\mathrm{K}$. Within the same symmetry, the first excited states, located $1.30 \mathrm{eV}$ above the ground state, is stable at short range with a $3 d^{2} 4 s$ electronic configuration. The asymptote of the ground state has a $3 \mathrm{~d} 4 \mathrm{~s}^{2}$ electronic configuration while those of the first excited states are $3 d^{2} 4 s$ and $3 d 4 s 4 p$, respectively. At a Sc-Bz distance of $10 \AA$, the latter are located 1.99 and $2.21 \mathrm{eV}$ above the ground state, compared with the experimental atomic excitations of 1.85 and $1.99 \mathrm{eV}$ respectively. The first excited ${ }^{2} \mathrm{E}_{2}$ state is quasidegenerate with a ${ }^{2} \mathrm{~A}_{1}$ state (see Table I). The potential energy curves for the quartet spin multiplicity are given in Fig. 4. The lowest state is found to have a ${ }^{4} \mathrm{~A}_{2}$ symmetry and lie at short range with a $3 \mathrm{de}_{2}{ }^{2} 4 \mathrm{~s}^{1}$ configuration. Several states with the ${ }^{4} \mathrm{~A}_{2}$ symmetry, are given in the Fig. 4 with $3 d^{2} 4 s^{1}$ or $3 d^{1} 4 s^{1} 4 p^{1}$ asymptotes. Close to the dissociation limit $(10 \AA)$, the $3 d^{1} 4 s^{1} 4 p^{1}$ configuration is located $0.48 \mathrm{eV}$ above the $3 \mathrm{~d}^{2} 4 \mathrm{~s}^{1}$ configuration, in very good agreement with the experimental atomic excitations of $0.51 \mathrm{eV}$. Avoided crossings involving several states occur within the $2.5-3.5 \AA$ range of the $\mathrm{Sc}-\mathrm{Bz}$ distance, resulting in a second minimum for the adiabatic ground state. Thus, the adiabatic curve for the ground state with a ${ }^{4} \mathrm{~A}_{2}$ symmetry presents two minima at 2.12 and $2.97 \AA$, respectively. They are separated by a barrier of $0.46 \mathrm{eV}$ on one side and $0.009 \mathrm{eV}$ on the other side. For more clearness, only the first of these two minima is reported in Table I. The relative position for all doublet and quartet states, bound at long or short range, are given in the Table I. The ground state is the ${ }^{2} \mathrm{E}_{2}$ state bound at long range, the ${ }^{4} \mathrm{~A}_{2}$ being $1.05 \mathrm{eV}$ above. 
TABLE I. Calculated relative energy, metal-benzene distance $\mathrm{d}$, binding energy (in $\mathrm{K}$ or eV) and dipole moment $\mu$ for MBz. The dipole moment is oriented from M to Bz.

\begin{tabular}{cccccc}
\hline \hline Molecule & State & Energy $(\mathrm{eV})$ & $\mathrm{d}(\AA)$ & Binding energy & $\mu(\mathrm{D})$ \\
\hline \multirow{2}{*}{ VBz } & ${ }^{4} \mathrm{E}_{2}$ & 0.00 & 4.82 & $350 \mathrm{~K}^{\mathrm{a}}$ & 0.60 \\
& ${ }^{6} \mathrm{~A}_{1}$ & 0.60 & 4.30 & $520 \mathrm{~K}^{\mathrm{a}}$ & 1.17 \\
& ${ }^{4} \mathrm{E}_{2}$ & 1.26 & 1.97 & $0.11 \mathrm{eV}^{\mathrm{a}, \mathrm{b}}$ & 2.79 \\
& ${ }^{2} \mathrm{E}_{2}$ & 1.70 & 1.91 & & 3.70 \\
& ${ }^{2} \mathrm{E}_{2}$ & 2.08 & 4.92 & & 0.67 \\
$\mathrm{ScBz}$ & 0.00 & 4.96 & $260 \mathrm{~K}^{\mathrm{a}}$ & 0.90 \\
& ${ }^{2} \mathrm{E}_{2}$ & 1.05 & 2.12 & $0.80 \mathrm{eV}^{\mathrm{a}}$ & 2.27 \\
& ${ }^{4} \mathrm{~A}_{2}$ & 1.26 & 2.12 & $1.19 \mathrm{eV}^{\mathrm{c}}$ & 3.15 \\
& ${ }^{2} \mathrm{~A}_{1}$ & 1.30 & 2.12 & $0.69 \mathrm{eV}^{\mathrm{c}}$ & 2.44 \\
& ${ }^{2} \mathrm{E}_{2}$ & 1.39 & 2.57 & $0.32 \mathrm{eV}^{\mathrm{c}}$ & 6.70 \\
& ${ }^{4} \mathrm{E}_{1}$ & 1.40 & 2.31 & $0.31 \mathrm{eV}^{\mathrm{c}}$ & 5.56 \\
& ${ }^{4} \mathrm{E}_{2}$ & 1.45 & 2.77 & $0.26 \mathrm{eV}^{\mathrm{c}}$ & 6.99 \\
& ${ }^{4} \mathrm{E}_{1}$ & 0.00 & 4.62 & $360 \mathrm{~K}^{\mathrm{a}}$ & 0.47 \\
& ${ }^{3} \mathrm{E}_{2}$ & 0.48 & 4.17 & $360 \mathrm{~K}^{\mathrm{a}}$ & 0.90 \\
& ${ }^{1} \mathrm{~A}_{1}$ & 0.78 & 1.62 & $0.14 \mathrm{eV}^{\mathrm{a}, \mathrm{b}}$ & 0.49 \\
\hline
\end{tabular}

MRCI+Davidson values.

${ }^{\mathrm{b}}$ Energy barrier for metastable states.

${ }^{\mathrm{c}} \mathrm{CASSCF}$ values.

\section{NiBz molecule}

For $\mathrm{NiBz}$, a singlet and a triplet states are found to be stable at long range with a binding energy of $360 \mathrm{~K}$. At short range, the singlet state is found to be metastable with respect to the dissociation into $\mathrm{Bz}+\mathrm{Ni}$ with an energy barrier of 0.14 $\mathrm{eV}$. The adiabatic singlet state has a $3 \mathrm{~d}^{8} 4 \mathrm{~s}^{2}$ electronic configuration at long range and a $3 \mathrm{~d}^{10}$ one at short range. The lowest triplet states are found to be repulsive at short range and the first short-range-lying triplet state presents a well at about $1.7 \mathrm{eV}$ above the doublet one.

\section{DISCUSSION}

The present results contrast with those obtained in previous calculations concerning both the spin multiplicity and the geometry of the ground state. The spin multiplicity obtained in our present CASSCF+MRCI study is compared with the previous calculations in Table II. For the three complexes considered, the ground state is found to be bound at

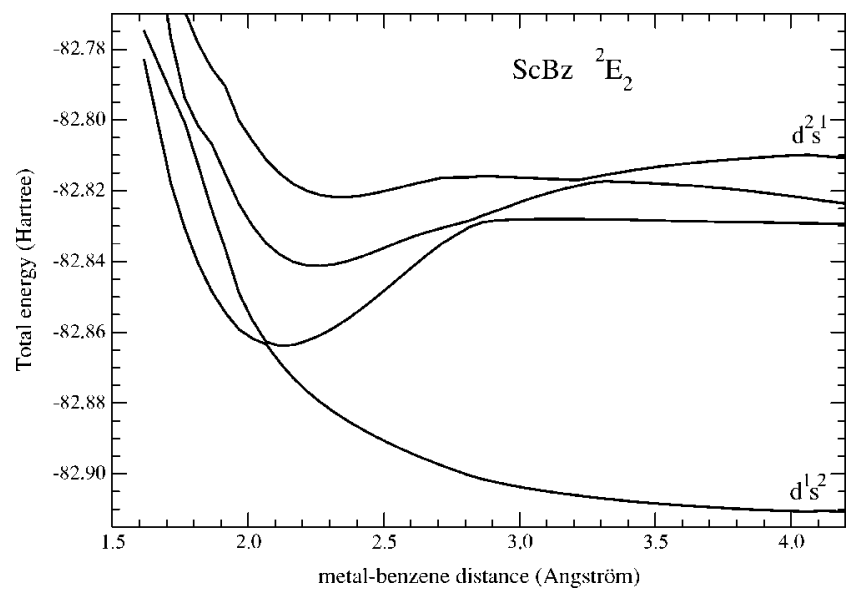

FIG. 3. Potential energy curves for the ${ }^{2} \mathrm{E}_{2}$ states for the $\mathrm{ScBz}$ molecule. long range in the present study. However, as the other studies investigated only short-range states, we also display in Table II the multiplicity obtained for the lowest state at short range in order to make a comparison. It should be noted that this short-range state is found to be only metastable with respect to the dissociation in metal atom and benzene molecule for the VBz and NiBz molecules. With DFT/BPW9I type calculations, result for the ground state of $\mathrm{VBz}$ was found to be very dependent on the basis set and the pseudopotential used. ${ }^{16,17}$ In a first study ${ }^{16}$ Pandey and co-workers found that the ground state is a sextet, corresponding to the state with the highest spin multiplicity, but in a recent study ${ }^{17}$ they found a quartet using the Lan $12 \mathrm{dz}$ pseudopotential and they found a doublet using an all-electrons $6-311 \mathrm{G}^{* *}$ basis set. Thus the DFT type calculations appear to be not able to distinguish between the different spin multiplicities of electronic states. In a study on the vertical electron-detachment energies of $\mathrm{ScBz}^{-}$, Kambalapalli and Ortiz ${ }^{10}$ have qualitatively investigated the structure of $\mathrm{ScBz}$ complexes at the

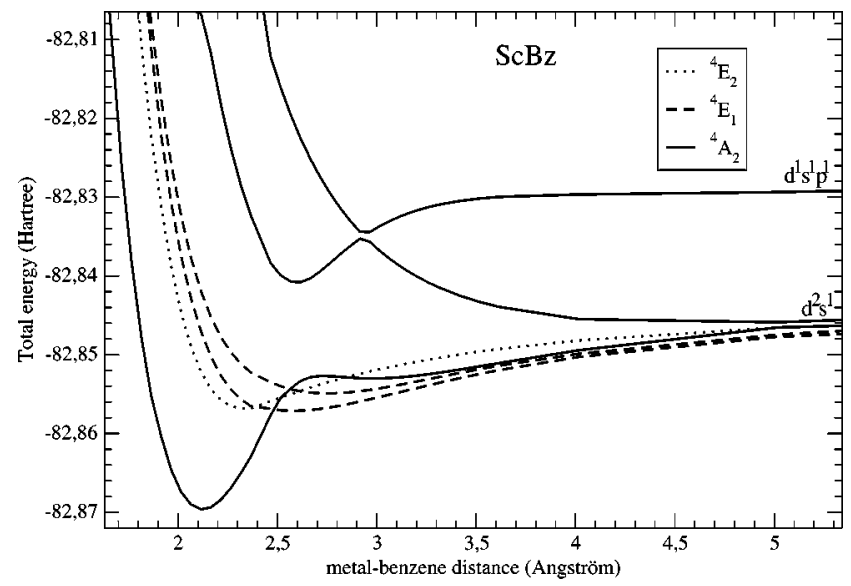

FIG. 4. Potential energy curves for the quartet states for the ScBz molecule. 
TABLE II. Spin multiplicity of the ground states obtained in the present CASSCF+MRCI study compared with results from previous works. In the latter the molecule lies at short range.

\begin{tabular}{|c|c|c|c|c|c|c|c|}
\hline \multirow[b]{2}{*}{ Molecule } & \multicolumn{2}{|c|}{$\mathrm{CASSCF}+\mathrm{MRCI}^{\mathrm{a}}$} & \multirow[b]{2}{*}{ BPW91/DNP ${ }^{b}$} & \multirow[b]{2}{*}{$\begin{array}{l}\text { BPW91/ } \\
\text { Lan12dz }\end{array}$} & \multirow[b]{2}{*}{$\begin{array}{l}\text { BPW91/ } \\
6-311 \mathrm{G}^{* * \mathrm{c}}\end{array}$} & \multirow[b]{2}{*}{$\begin{array}{c}\text { MP2/ } \\
6-311 \mathrm{G}^{\mathrm{d}}\end{array}$} & \multirow[b]{2}{*}{$\mathrm{FVCI}^{\mathrm{e}}$} \\
\hline & $\begin{array}{c}\text { Stable } \\
\text { long-range state }\end{array}$ & $\begin{array}{l}\text { Stable or metastable } \\
\text { short-range state }\end{array}$ & & & & & \\
\hline $\mathrm{VBz}$ & 4 & $4^{\mathrm{f}}$ & 6 & 4 & 2 & & $4^{\mathrm{f}}$ \\
\hline $\mathrm{ScBz}$ & 2 & 4 & 4 & & & 2 & \\
\hline $\mathrm{NiBz}$ & 3 & $1^{\mathrm{f}}$ & 1 & & & & \\
\hline 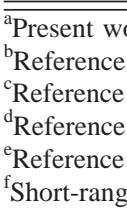 & $\begin{array}{l}\text { ork. } \\
16 . \\
17 . \\
10 . \\
15 . \\
\text { ge metastable state }\end{array}$ & & & & & & \\
\hline
\end{tabular}

UHF + MP2 level (UHF-unrestricted Hartree-Fock). They found a doublet that displays a $\mathrm{C}_{2} \mathrm{v}$ boat conformation for the ground state, $\mathrm{a}_{6} \mathrm{v}$ quartet lying $0.1 \mathrm{eV}$ above it. However, the authors specified that the calculations on doublets produce unacceptably large spin contamination, and the corresponding relative energies could not be considered reliable. Finally, Yasuike et al. ${ }^{15}$ using a full valence configuration interaction (FVCI) method, have investigated the potential energy profile of the VBz complex. They found that the lowest lying state is a short-range quartet metastable with respect to the dissociation in $\mathrm{V}+\mathrm{Bz}$ molecule, similarly to the present results. The long-range states were not considered in their study.

From all these above results, it appears clearly that the characterization of the ground-state spin multiplicity is very dependent on the method of calculation. Theory has difficulties to treat the complexity of the electronic structure of transition-metal-benzene systems due to the occurrence of low-lying metal excited states that can mix with the ground state or compete with it for the most stable bonding configuration. These systems have many spin-multiplet symmetries lying within a narrow energy range that the calculations are not able to distinguish. Although the present multireference calculations are expected to be more reliable than single determinantal approaches to investigate the potential energy surfaces presenting some crossing regions (see discussion on the treatment of avoided crossing in DFT calculations in Ref. $25)$, it is difficult to conclude definitively on the spin multiplicity of the ground state.

Unfortunately there have been no experimental reports in the gas phase concerning the magnetic dipole measurements which would allow a direct experimental probe of the spin multiplicity. The only available experimental results on neutral $\mathrm{MBz}$ complexes are the studies by Dugourd and co-workers, ${ }^{26}$ and independently by Imura, Ohoyama and Kasai, ${ }^{27}$ concerning the electric dipole moment. These experimental data can be compared with the present calculated values, given in Table I, in order to confirm the geometry of the ground state. Indeed, the dipole moment of the longrange states are inferior than $1 \mathrm{D}$, while those of short-range states are much larger. The experimentalists found, respectively, a dipole of $0.17 \pm 0.3 \mathrm{D}$ (Ref. 26) and 1.0 $\pm 0.3 \mathrm{D}$ (Ref. 27) for $\mathrm{VBz}$ and $0.7 \pm 0.3 \mathrm{D}$ (Ref. 26) and a value $<0.6 \mathrm{D}$
(Ref. 27) for NiBz. These data compare well with the present calculated values of 0.60 and $0.47 \mathrm{D}$ for the long-range states of $\mathrm{VBz}$ and $\mathrm{NiBz}$, respectively, but they are not consistent with the theoretical values obtained for the short-range states of $\mathrm{VBz}(2.79 \mathrm{D})$. For ScBz, Dugourd and co-workers found $1.6 \pm 0.3 \mathrm{D}$ in quite satisfactory agreement with the calculated value of $0.90 \mathrm{D}$ for the long-range state. Thus, comparisons with the experimental data are in favor of longrange states. The existence of these states have been recently confirmed in a theoretical study using a long range model, adding a repulsive term to an attractive dispersion term estimated in an oblate-spheroidal approach. ${ }^{28}$ However, the calculated binding energies of such long-range states, about 300 $\mathrm{K}$, are small and perhaps under-estimated. Theoretical investigations using other methods taking into account electronic correlationlike multireference perturbation methods could be envisaged to characterize the long-range states as they are expected to give correct binding energies.

\section{CONCLUSION}

We have presented an $a b$ initio study of the geometry and electronic properties of the transition-metal-benzene complexes $\mathrm{ScBz}, \mathrm{VBz}$, and NiBz. For each complex, the lowest energy molecular state was found to be a long-range state. Such long-range states were seen to be compatible with recent dipole moment measurements. Present ab initio calculations and previous DFT type calculations lead to contradictory results concerning both the geometry and the spin multiplicity of the ground state. Theoretical investigations should be completed by the use of other methods taking into account electronic correlationlike multireference perturbation methods to confirm our present conclusions. We hope that present theoretical results would stimulate experimental investigations on those complexes, such as magnetic dipole measurements which would allow a direct experimental probe of the spin multiplicity, but have not been performed yet.

\section{ACKNOWLEDGMENT}

This work benefited from allocation of computer resources at the Institut du Developpement et des Ressources en Informatique Scientifique (IDRIS) du Centre National de la Recherche Scientifique, France. 
${ }^{1}$ A. Nakajima and K. Kaya, J. Phys. Chem. A 104, 176 (2000).

${ }^{2}$ T. Kurikaya, H. Takeda, M. Hirano, K. Judai, T. Arita, S. Nagao, A. Nakajima, and K. Kaya, Organometallics 18, 1430 (1999).

${ }^{3}$ P. Weis, P. R. Kemper, and M. T. Bowers, J. Phys. Chem. A 101, 8207 (1997).

${ }^{4}$ D. Rayane, A. R. Allouche, R. Antoine, M. Broyer, I. Compagnon, and P. Dugourd, Chem. Phys. Lett. 375, 506 (2003).

${ }^{5}$ C. W. Bauschlicher, H. Partridge, and S. R. Langhoff, J. Phys. Chem. 96, 3273 (1992).

${ }^{6}$ C. N. Yang and S. J. Klippenstein, J. Phys. Chem. A 103, 1094 (1999).

${ }^{7}$ P. Chaquin, D. Costa, C. Lepetit, and M. Che, J. Phys. Chem. A 105, 4541 (2001).

${ }^{8}$ J. Molina Molina, J. A. Dobado, and S. Melchor, J. Mol. Struct. 589-590, 337 (2002).

${ }^{9}$ T. D. Jaeger, D. Van Heijnsbergen, S. J. Klippenstein, G. Von Helden, G. Meijer, and M. A. Duncan, J. Am. Chem. Soc. 126, 10981 (2004).

${ }^{10}$ S. Kambalapalli and J. V. Ortiz, J. Phys. Chem. A 108, 2988 (2004)

${ }^{11}$ T. Yasuike and S. Yabushita, J. Phys. Chem. A 103, 4533 (1999).

${ }^{12}$ G. E. Froudakis, A. N. Andriotis, and M. Menon, Chem. Phys. Lett. 350, 393 (2001).

${ }^{13}$ B. K. Rao and P. Jena, J. Chem. Phys. 116, 1343 (2002).

${ }^{14}$ B. K. Rao and P. Jena, J. Chem. Phys. 117, 5234 (2002).

${ }^{15}$ T. Yasuike, A. Nakajima, S. Yabushita, and K. Kaya, J. Phys. Chem. A 101, 5360 (1997).
${ }^{16}$ R. Pandey, B. K. Rao, P. Jena, and M. Alvarez Blanco, J. Am. Chem. Soc. 123, 3799 (2001).

${ }^{17}$ A. K. Kandalam, B. K. Rao, P. Jena, and R. Pandey, J. Chem. Phys. 120, 10414 (2004).

${ }^{18}$ S. Dennler, J. Morillo, G. M. Pastor, Surf. Sci. 532-535, 334 (2003), and references therein.

${ }^{19}$ MOLPRO is a package of ab initio programs written by H. J. Werner and P. J. Knowles, with contributions from R. D. Amos, A. Berning, D. L. Cooper et al., University of Birmingham, 2000.

${ }^{20}$ Gabedit is a graphical user interface for MOLPRO available from http:// lasim.univlyon 1.fr/allouche/gabedit

${ }^{21}$ M. Dolg, U. Wedig, H. Stoll, and H. Preuss, J. Chem. Phys. 86, 866 (1987).

${ }^{22}$ G. Igel-Mann, H. Stoll, and H. Preuss, Mol. Phys. 65, 1321 (1988).

${ }^{23}$ W. J. Stevens, M. Krauss, H. Basch, and P. G. Jasien, Can. J. Chem. 70, 612 (1992).

${ }^{24}$ W. J. Stevens, H. Basch, and M. Krauss, J. Chem. Phys. 81, 6026 (1984).

${ }^{25}$ S. G. Wang and W. H. E. Schwarz, J. Chem. Phys. 105, 4641 (1996).

${ }^{26}$ F. Rabilloud, D. Rayane, A. R. Allouche, R. Antoine, M. Aubert-Frécon, M. Broyer, I. Compagnon, and P. Dugourd, J. Phys. Chem. A 107, 11347 (2003).

${ }^{27}$ K. Imura, H. Ohoyama, and T. Kasai, Chem. Phys. 301, 183 (2004).

${ }^{28}$ M. Aubert-Frécon, F. Rabilloud, and A. R. Allouche, Chem. Phys. Lett. (to be published). 